\title{
Patterns of inflammatory responses and parasite tolerance vary with malaria transmission intensity
}

\author{
Temitope W. Ademolue ${ }^{1}$, Yaw Aniweh¹, Kwadwo A. Kusi² and Gordon A. Awandare ${ }^{1,2^{*}}$ (1)
}

\begin{abstract}
Background: In individuals living in malaria-endemic regions, parasitaemia thresholds for the onset of clinical symptoms vary with transmission intensity. The mechanisms that mediate this relationship are however, unclear. Since inflammatory responses to parasite infection contribute to the clinical manifestation of malaria, this study investigated inflammatory cytokine responses in children with malaria from areas of different transmission intensities (ranging from low to high).

Methods: Blood samples were obtained from children confirmed with malaria at community hospitals in three areas with differing transmission intensities. Cytokine levels were assessed using the Luminex ${ }^{\circledR}$-based magnetic bead array system, and levels were compared across sites using appropriate statistical tests. The relative contributions of age, gender, parasitaemia and transmission intensity on cytokine levels were investigated using multivariate regression analysis.

Results: Parasite density increased with increasing transmission intensity in children presenting to hospital with symptomatic malaria, indicating that the parasitaemia threshold for clinical malaria increases with increasing transmission intensity. Furthermore, levels of pro-inflammatory cytokines, including tumour necrosis factor alpha (TNF-a), interferon-gamma (IFN- $\gamma$ ), interleukin (IL)-1 $\beta, I L-2, I L-6, I L-8$, and IL-12, decreased with increasing transmission intensity, and correlated significantly with parasitaemia levels in the low transmission area but not in high transmission areas. Similarly, levels of anti-inflammatory cytokines, including IL-4, IL-7, IL-10 and IL-13, decreased with increasing transmission intensity, with IL-10 showing strong correlation with parasitaemia levels in the low transmission area. Multiple linear regression analyses revealed that transmission intensity was a stronger predictor of cytokine levels than age, gender and parasitaemia.
\end{abstract}

Conclusion: Taken together, the data demonstrate a strong relationship between the prevailing transmission intensity, parasitaemia levels and the magnitude of inflammatory responses induced during clinical malaria.

Keywords: Cytokines, Immunity, Malaria, Parasite tolerance, Transmission intensity

\section{Background}

In endemic areas, protection against clinical malaria results from repeated exposure to Plasmodium falciparum parasites $[1,2]$, such that individuals residing in

\footnotetext{
*Correspondence: gawandare@ug.edu.gh

${ }^{1}$ West African Center for Cell Biology of Infectious Pathogens, Department of Biochemistry, Cell and Molecular Biology, College of Basic and Applied Sciences, University of Ghana, Legon, Accra, Ghana Full list of author information is available at the end of the article
}

holo-endemic areas can tolerate high levels of parasites without showing clinical symptoms. In low transmission areas however, clinical malaria has been associated with low parasite thresholds [3], suggesting that the threshold parasitaemia for clinical malaria differs in children of similar ages who reside in areas with different transmission intensities [4-6]. These patterns demonstrate that the mechanisms of anti-parasite immunity are distinct from those responsible for anti-disease immunity or parasite tolerance. 
Increase in the breadth and magnitude of parasitespecific antibody responses following repeated parasite exposures [7] is expected to control parasitaemia, and reduce the incidence of clinical disease [8]. However, this is not always true in high transmission areas, where children could harbour relatively high parasitaemia but remain asymptomatic $[1,2,7]$. Therefore, while adaptive immune responses may adequately account for anti-parasite immunity, the mechanisms for anti-disease immunity or parasite tolerance remain unclear.

Clues to the mechanisms of parasite tolerance may lie in the role of inflammatory cytokines, which have been shown to correlate with the onset of symptomatic disease during P. falciparum infection [9-15]. Plasmodium falciparum infection causes paroxysmal fever that is triggered by strong pro-inflammatory responses involving pyrogenic cytokines such as interleukin (IL)-1 $\beta$ and tumour necrosis factor alpha (TNF- $\alpha$ ) [16]. Although inflammatory responses, including interferon gamma (IFN- $\gamma$ ), IL-12, IL-1 $\beta$, IL-2, and TNF$\alpha$, play important roles that facilitate parasite clearance $[9$, 17, 18], circulating high levels of these cytokines have been associated with malaria immunopathology $[11,12,14,19-$ 23]. Similarly, high levels of pro-inflammatory cytokines released during malaria infection have been associated with several pathologic processes such as sequestration of infected red blood cells (iRBCs) [24, 25], organ-specific inflammation that results in complications such as cerebral malaria [15, 26, 27], and placental malaria [28]. To prevent these deleterious effects, anti-inflammatory cytokines such as IL-10, IL-4, IL-17, and IL-13 are secreted to balance the effects of pro-inflammatory cytokines $[29,30]$.

The intensity of transmission has been shown to be a major predictor of clinical manifestations and outcomes of malaria in endemic areas $[6,31]$. In holo-endemic areas, disease severity is predominantly related to hyperparasitaemia and severe malarial anaemia $[6,31,32]$, whereas in low to medium transmission areas, there is a high rate of cerebral malaria [6,31,33,34]. Given the importance of pro-inflammatory mediators in determining manifestations of malaria, this study investigated the relationship between transmission intensity and inflammatory cytokine responses in children with symptomatic malaria. The roles of these factors in influencing the levels of parasitaemia were also examined. The results provide evidence of a strong relationship between transmission intensity and inflammatory responses during acute malaria infection, and suggest that these factors influence the levels of parasitaemia at clinical presentation.

\section{Methods}

\section{Study sites}

Three outpatient hospitals at locations (Kintampo, Navrongo and Accra) representing distinct malaria transmission intensities in Ghana were selected for this study. Kintampo is holo-endemic for malaria with yearround transmission, and an entomological inoculation rate (EIR) of $>250$ infective bites/person/year [35]. Navrongo is hyperendemic for malaria with seasonal rainfall and transmission (high transmission from May to November, low transmission from December to April) and EIR of 50-250 infective bites/person/year [36]. Accra is the capital city and has a relatively low transmission intensity $(<50$ infective bites/person/year) that peaks between June and August annually [37]. Samples were collected from 2011 to 2013 during the peak transmission seasons at the respective study sites.

\section{Participants and sample collection}

Ethical approvals were obtained from the ethics committees of the Ghana Health Service, Navrongo Health Research Centre, Kintampo Health Research Centre and Noguchi Memorial Institute for Medical Research, University of Ghana, Accra, Ghana. Participation was voluntary, and written informed consent was obtained from parents/guardians of the children. Study participants were children aged 2-14 years who were showing signs of clinical malaria, and had been referred for malaria tests by the attending physician. Parasitaemia was detected by malaria rapid diagnostic tests (RDTs) and confirmed by microscopic examination of thick and thin blood smears. Parasite density was estimated by counting the number of parasites per 200 white blood cells as previously described [38, 39]. Haemoglobin levels were quantified using an automated haematology analyzer. Before delivery of anti-malarial and/or any other treatment interventions, $5 \mathrm{~mL}$ of venous blood was obtained from each child. Plasma samples were separated from whole blood by centrifugation at $2500 \mathrm{rpm}$ (Eppendorf, model: $5810 \mathrm{R}$ ) for $10 \mathrm{~min}$ and aliquoted into Eppendorf tubes for storage at $-80{ }^{\circ} \mathrm{C}$ until further experiments. Sample collection, storage and analysis were done using the same protocols and procedures to ensure uniformity and comparability of data from the different hospitals.

\section{Cytokine assays}

Plasma concentrations of cytokines were measured using the highly sensitive xMAP technology (Luminex Corporation), which allows the simultaneous quantification of several biological analytes in a 96-well format. The MILLIPLEX $^{\circledR}$ MAP 13-Plex Kits from Millipore (Merck Group, magnetic beads) were used because of their higher detection accuracy and reproducibility of results compared to other vendors [40]. These kits were used to quantify eight pro-inflammatory cytokines (TNF- $\alpha$, IFN$\gamma$, IL-1 $\beta$, IL-2, IL-8, granulocyte monocyte colony stimulating factor (GM-CSF), IL-6 and IL-12p70) and four 
anti-inflammatory cytokines (IL-4, IL-7, IL-10, IL-13) in duplicate wells for each plasma sample. These analytes were selected based on relevance and association with malaria. The assays were conducted strictly following the manufacturer's instructions without any modification. The kits used were from the same lot, and the samples were randomly distributed across plates. Prior to assay, samples were thawed and clarified by centrifugation (2000 rpm for $10 \mathrm{~min}$ ). There were no readings from the background wells while the quality control and the Standards wells were within the specified range of the kits. Samples with percentage coefficient of variation $(\% \mathrm{CV})>15 \%$ were excluded from further analysis. Cytokine detection limits are found in Additional file 1.

\section{Statistical analyses}

Data analyses and graphs were done using GraphPrism version 6.01 (GraphPad Software, Inc.) and Minitab version 17.1.0.0 (Minitab Inc.). After initial normality tests, patients' demographics and clinical parameters were compared across the three sites either by Pearson's Chi square $\left(x^{2}\right)$ test (to compare proportions in categorical variables) or One-way ANOVA or Kruskal-Wallis $\mathrm{H}(\mathrm{K}-\mathrm{W})$ test (for continuous data sets), depending on normality of data. An across-site comparison of cytokine levels was performed with the K-W test, while Dunn's multiple comparison test was used to reveal between-site pairwise significant differences. Spearman's correlation analyses were performed for associations of cytokines levels with age and parasite density. In addition, a Spearman's correlation matrix was built to detect associations between cytokines. Multiple linear regression analyses were conducted to detect the variable(s) that is/are the best predictor(s) of cytokine levels. For the regression models, cytokine levels served as the outcome variables while parasitaemia, age, gender and transmission intensity served as the predictor variables. Statistical significance was generally set at $P<0.05$, however, after Bonferroni's procedure, the critical value $(\alpha)$ of the regression models was adjusted to 0.004 .

\section{Results \\ Demographic and clinical characteristics of patients across the study sites}

To investigate the role of inflammatory responses in the development of malaria parasite tolerance in endemic areas, this study examined the relationship between transmission intensity and the patterns of cytokine production in children with malaria. Using a cross-sectional approach, a total of 173 children who tested positive for malaria by RDTs and microscopy were recruited from three community hospitals in three areas with varying transmission intensities: Accra $(N=71)<$ Navrongo $(N=44)<$ Kintampo $(N=58)$. The proportions of both sexes were comparable across the study sites $(P=0.270$; Table 1). Children in Accra were relatively older than those in Navrongo $(P=0.010)$ and Kintampo $(P=0.025)$, however, the ages of children from Navrongo and Kintampo did not differ $(P=0.999)$. Parasitaemia reflected the intensity of transmission, with children from Kintampo having higher parasitaemia compared with those in Accra $(P=0.005)$ and Navrongo $(P=0.070)$, although these differences were not statistically significant for Navrongo (Table 1; Additional file 2). Haemoglobin levels decreased as transmission intensity increased $(P=0.007)$, with children from Accra having significantly higher haemoglobin levels compared with those from Kintampo $(P=0.008)$. Although haemoglobin levels in children in Accra were also higher than those in Navrongo, this difference was not statistically significant $(P=0.076)$. Children from Navrongo and Kintampo had comparable haemoglobin levels $(P=0.545)$. The median temperature at clinic also decreased with increasing transmission intensity $(P<0.001)$, with children from

Table 1 Demographic and clinical parameters of patients across the study sites

\begin{tabular}{lllll}
\hline Characteristics & Accra & Navrongo & Kintampo & $P$ value \\
\hline Participants (number) & 71 & 44 & 58 & - \\
Female (number, \%) & 35.3 & 50.0 & 43.2 & $0.270^{\mathrm{b}}$ \\
Median age (IQR), years & $6(4-9)$ & $4(3-6)$ & $4(2-6)$ & $0.005^{\mathrm{a*}}$ \\
Median parasitemia (IQR), per $\mu \mathrm{L}$ & $21,805(7,172-64,355)$ & $46,351(18,524-66,679)$ & $70,215(11,342-209,335)$ & $0.004^{\mathrm{a} *}$ \\
Mean hemoglobin level (IQR), g/dL & $10.5(9.0-11.6)$ & $9.8(8.8-10.9)$ & $9.6(8.1-11.2)$ & $0.007^{\mathrm{c*}}$ \\
Median temperature (IQR), ${ }^{\circ} \mathrm{C}$ & $38.8(38.0-39.4)$ & $38.0(37.0-39.0)$ & $37.1(36.5-38.3)$ & $0.0001^{\text {a* }}$ \\
\hline
\end{tabular}

IQR interquartile range

*Significant difference

a Kruskal-Wallis $\mathrm{H}$ test

${ }^{b} x^{2}$ test

c One-way ANOVA 
Accra having significantly higher median temperature compared to Navrongo $(P<0.001)$ and Kintampo $(P<0.001)$. Children in Navrongo also had higher median temperature than those from Kintampo $(P<0.001)$. Since these children were recruited at presentation to hospital with symptoms of malaria, the pattern of parasitaemia suggests that the parasitaemia threshold for clinical manisfestation of infection seems to increase with increasing transmission intensity.

\section{Levels of pro-inflammatory mediators decline with increasing transmision intensity}

The secretion of pro-inflammatory cytokines during malaria infection has been shown to culminate in the clinical manifestations of disease [13, 14]. Consequently, the role of cytokine levels in mediating parasite tolerance was investigated in children exposed to different malaria transmission intensities. Quantification of levels of pro-inflammatory cytokines, including TNF- $\alpha$, IFN- $\gamma$, IL-1 $\beta$, IL-2, IL-8, IL-6, IL-12, and GM-CSF in children with malaria in the three transmission areas revealed a pattern of decreasing cytokine levels with increasing transmission intensity (Accra > Navrongo > Kintampo; Fig. 1). Levels of all pro-inflammatory cytokines were significantly lower in children from Kintampo compared to those in Accra $(P<0.005$ for all cytokines; Fig. 1a-h). In addition, levels of all pro-inflammatory cytokines except IFN- $\gamma(P=0.834)$, IL-8 $(P=0.056)$ and IL-6 $(P=0.260)$ were lower in the Kintampo group compared to the Navrongo group $(P<0.05$ for all comparisons; Fig. 1$)$. Given that parasitaemia levels increased with increasing transmission intensity (Table 1), the reverse pattern of pro-inflammatory cytokine suggest that lower cytokine responses appear to favour increased parasite tolerance.

\section{Levels of anti-inflammatory mediators also decline with increasing transmission intensity}

Since pro-inflammatory responses are usually followed by the secretion of anti-inflammatory mediators to balance their effects $[13,14,29,30]$, the levels of four key anti-inflammatory cytokine including IL-4, IL-7, IL-10, and IL-13 were also examined. The pattern observed was similar to that of the pro-inflammatory cytokines, with cytokine levels decreasing with increasing transmission intensity across the three areas
(Accra > Navrongo > Kintampo; Fig. 2). Specifically, levels of all four cytokines were significantly lower in children recruited in Kintampo compared to those in Accra $(P<0.01$ for all comparisons; Fig. 2$)$. Taken together, these data buttress the patterns observed for pro-inflammatory responses, and further confirm an association between transmission intensity and the magnitude of inflammatory responses induced during clinical malaria, and suggest a likely association with parasitaemia levels.

\section{Correlation between parasitaemia and pro-infammatory cytokine levels varies with transmission intensites}

Given the strong relationship between cytokine levels and transmission intensity, the correlations between levels of pro-inflammatory mediators and parasitaemia were directly examined in each of the three sites. There were significant positive correlations between parasite density in children with malaria and levels of key pro-inflammatory cytokines, including TNF- $\alpha$, IFN- $\gamma$ and IL-6 (Fig. 3). However, these correlations were observed in the Accra group only, with none showing significant correlation in the Navrongo group, and only IL-6 showing a significant correlation in the Kintampo group (Fig. 3). Since malaria parasite antigens induce the production of pro-inflammatory cytokines $[14,16,41,42]$, these results are further evidence of increased parasite tolerance in the higher transmission areas, where further increases in parasite levels above a certain threshold no longer augment cytokine production.

\section{Limited correlations between parasitemia and anti-inflammatory cytokine levels}

Subsequently, the correlations between anti-inflammatory cytokines and parasite burden were examined in children with malaria across the different transmission areas. Unlike the patterns observed with the pro-inflammatory cytokines, the anti-inflammatory cytokines did not generally show significant correlation with parasitaemia in any of the endemic areas (Fig. 4). However, IL-10, which is considered a critical anti-inflammatory mediator in regulating the pro-inflammatory response during malaria [29, 43, 44], showed significant correlation with parasite density in children residing in Accra and Kintampo (Fig. 4). This correlation was particularly strong in the Accra group, which is consistent with the correlation

\footnotetext{
(See figure on next page.)

Fig. 1 Pattern of pro-inflammatory responses to malaria infection across different transmission sites. Plasma levels of pro-inflammatory cytokines a tumour necrosis factor (TNF)- $a, \mathbf{b}$ interferon (IFN)- $\gamma, \mathbf{c}$ interleukin (IL)-2, $\mathbf{d}\|\mathrm{L}-1 \beta, \mathbf{e}\| \mathrm{L}-12, \mathbf{f}\|\mathrm{L}-6, \mathbf{g}\| \mathrm{L}-8$, and $\mathbf{h}$ granulocyte macrophage colony stimulating factor (GM-CSF), were quantified in children with malaria in three areas of Ghana with varying malaria transmission intensities (Accra $<$ Navrongo < Kintampo). Comparisons across sites were performed using Kruskal-Wallis $\mathrm{H}$ test with Dunn's posthoc test (Accra $N=71 ; \mathrm{Navrongo} N$ $=44$; Kintampo $N=58$ ). Data are presented as box plots where boxes represent the inter-quartile ranges, while the whiskers represent the 10th and 90th percentiles. The lines across the boxes indicate the median values, while closed circles represent outliers
} 

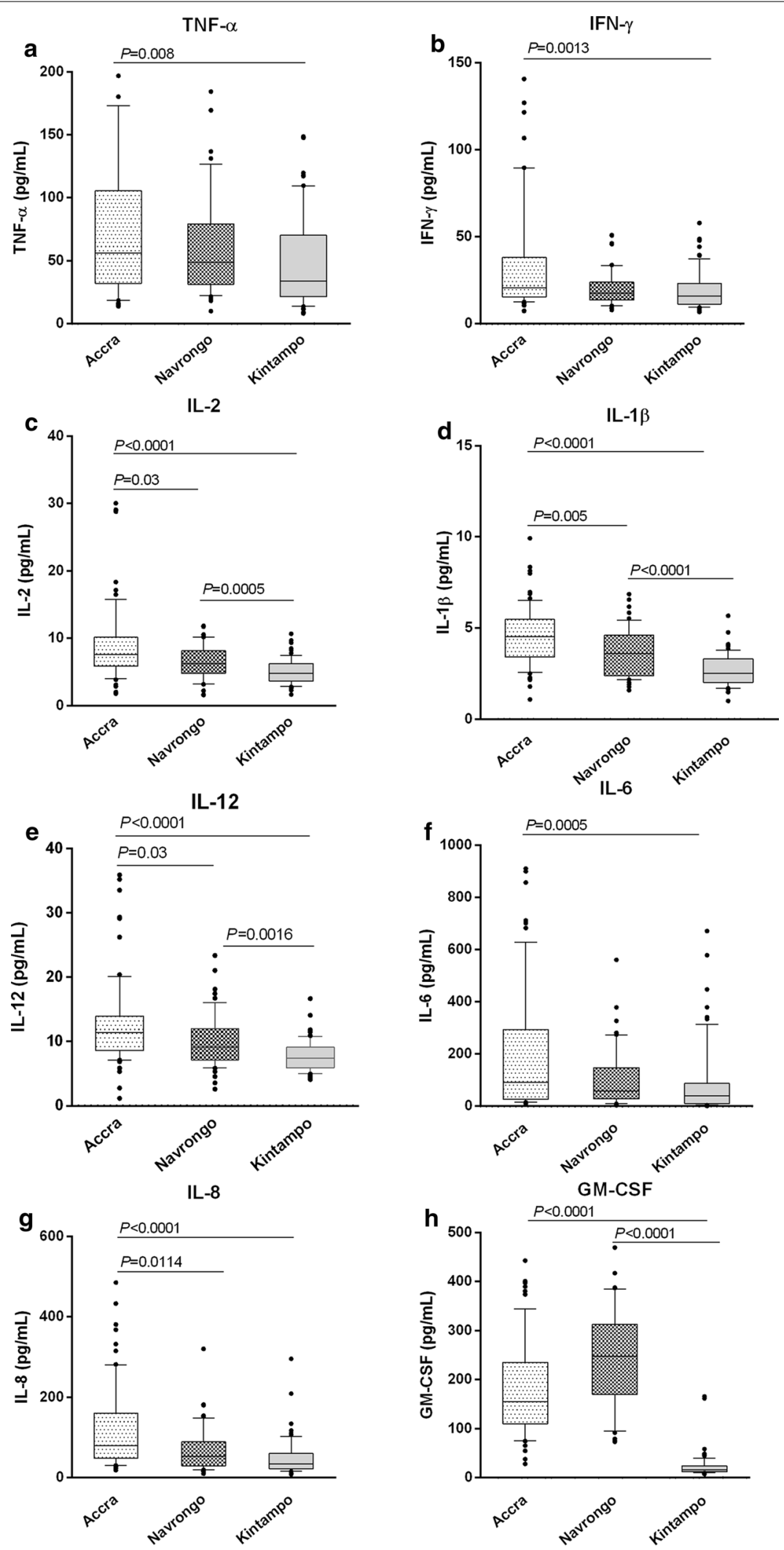

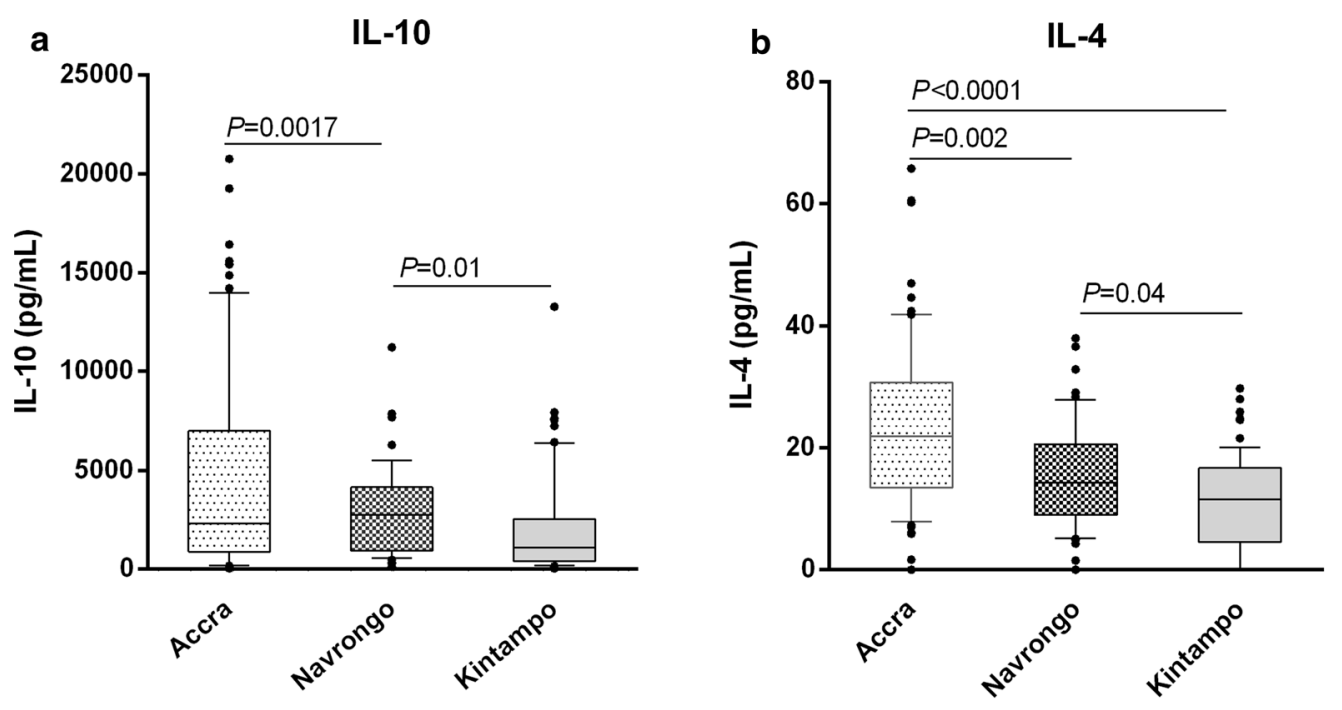

\section{IL-13}

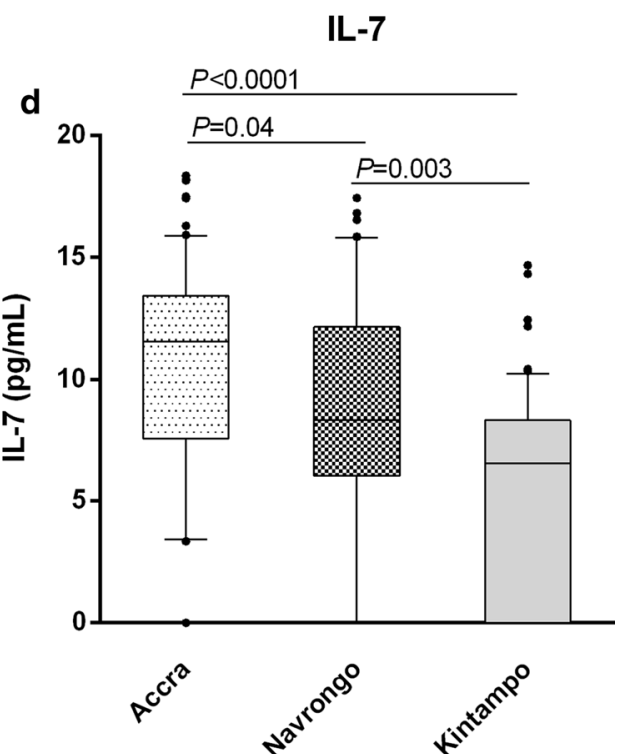

Fig. 2 Pattern of anti-inflammatory responses to malaria infection across the different transmission areas. Plasma levels of anti-inflammatory cytokines a interleukin (IL)-10, b IL-4, $\mathbf{c} \mid \mathrm{L}-13$, and $\mathbf{d} \mid \mathrm{L}-7$, were quantified in children with malaria in three areas of Ghana with varying malaria transmission intensities (Accra < Navrongo < Kintampo). Comparisons across sites were performed using Kruskal-Wallis $\mathrm{H}$ test with Dunn's posthoc test (Accra $N=71$; Navrongo $N=44$; Kintampo $N=58$ ). Samples below the detection limits were assigned a concentration of zero, including 12 samples for IL4 (Accra = 3, Navrongo = 1, Kintampo = 8), and 33 samples for IL7 (Accra =6, Navrongo = 7, Kintampo $=20)$. Data are presented as box plots where boxes represent the inter-quartile ranges, while the whiskers represent the 10th and 90th percentiles. The lines across the boxes indicate the median values, while closed circles represent outliers

(See figure on next page.)

Fig. 3 Association between pro-inflammatory cytokines and parasite density across the sites. The relationships between parasite density in children with malaria and plasma levels of pro-inflammatory cytokines $\mathbf{a}$ tumour necrosis factor (TNF)- $a \mathbf{b}$ interleukin (IL)-12, $\mathbf{c}$ interferon (IFN)- $\gamma$, d IL-1 $\beta, \mathbf{e}$ $\mathrm{IL}-2, \mathbf{f} \mathrm{IL}-6$ and $\mathbf{g} \mathrm{LL}-8$, were examined using Spearman's rank correlation test. $P$ values in bold type indicate statistical significance. Samples below the detection limits were excluded from the analysis. ( $\rho=$ Spearman's correlation coefficient) 


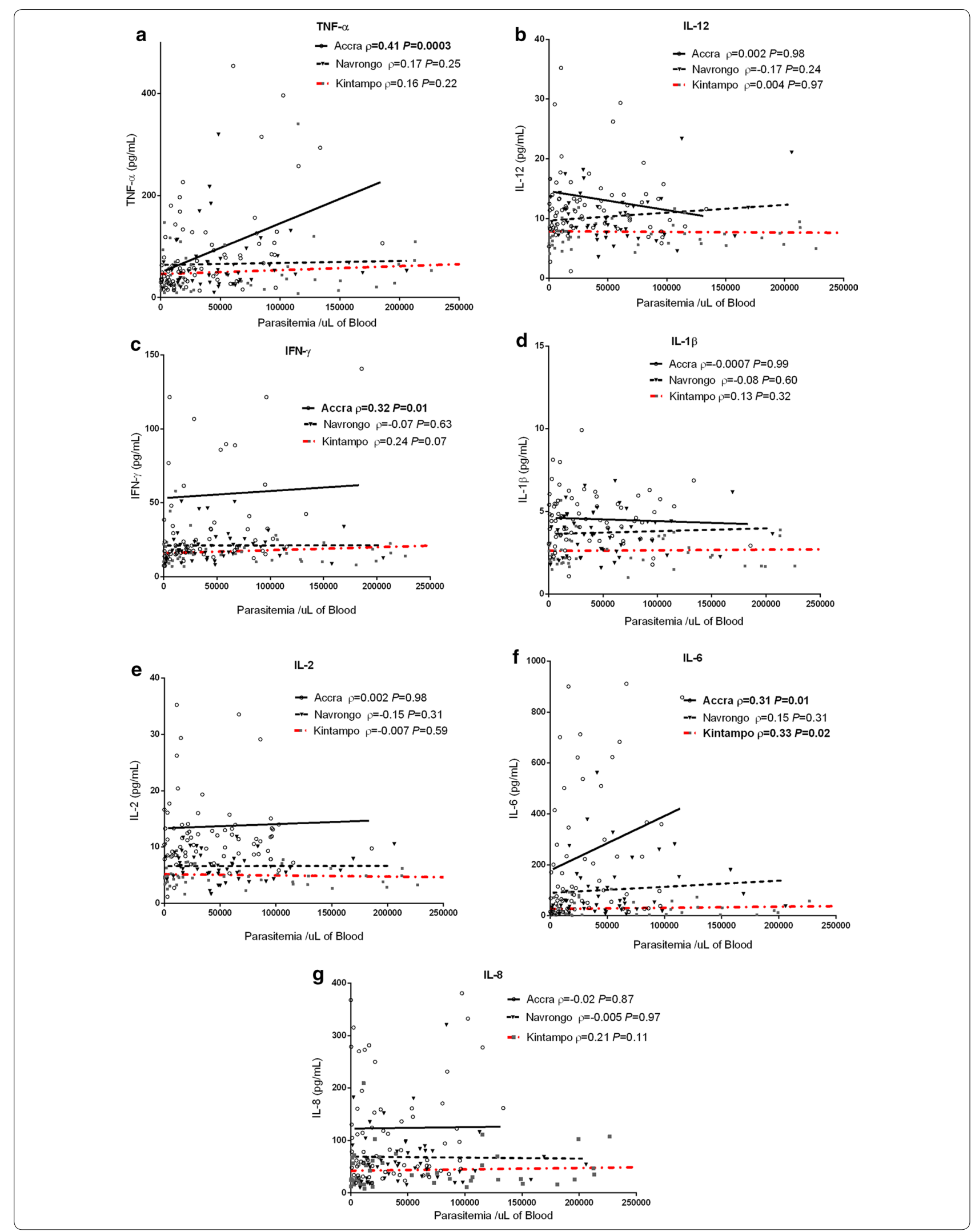



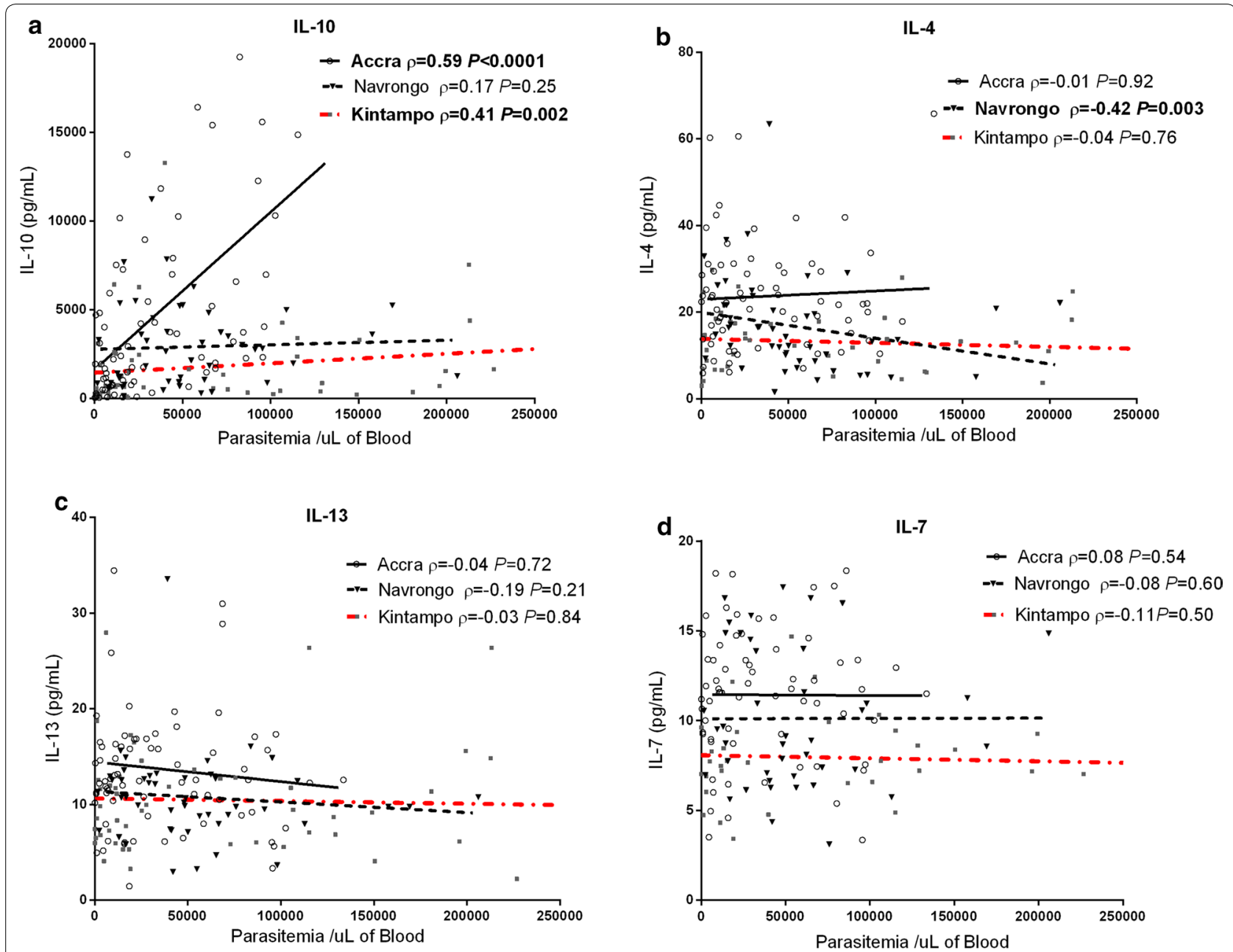

Fig. 4 Association between anti-inflammatory cytokines and parasite density across the sites. The relationships between parasite density in children with malaria and plasma levels of anti-inflammatory cytokines a interleukin (IL)-10, b $\mid L-4, \mathbf{c} \| \mathrm{L}-13$, and $\mathbf{d} \| \mathrm{L}-7$, were examined using Spearman's rank correlation test. $P$ values in bold type indicate statistical significance. Samples below the detection limits were excluded from the analysis. $(\rho=$ Spearman's correlation coefficient)

observed for the key pro-inflammatory cytokines. In addition, IL-4 showed a significant association with parasitaemia in children from Navrongo, however, this correlation was negative (Fig. 4).

\section{Associations between parasite density and cytokine levels are independent of age across sites}

Cytokine levels during parasitic infections, including malaria, have been shown to vary with age [45]. Since age significantly differed between sites (Table 1), the study subsequently determined whether the age difference affected cytokine responses during acute malaria infection. The results showed limited associations between age and cytokine levels in this cohort, with IFN- $\gamma$ correlating negatively with age in Navrongo and Kintampo (Additional file 3), while IL-8 and IL-4 showed positive correlations with age in Navrongo and Accra, respectively (Additional file 3). Thus, contrary to previous reports where age was found to significantly affect levels of cytokines during malaria infection $[6,13,45,46]$, age did not seem to be a major determinant of plasma levels of cytokines across the sites.

\section{Transmission intensity is the major predictor of cytokine responses}

Using multiple linear regression analyses, the relative contributions of age, gender, parasite density, and transmission intensity as predictors of cytokine levels was examined. These analyses revealed that study site (coded in order of increasing transmission intensity) was the strongest predictor of all cytokine levels, except GMCSF, for which sex was the best predictor (see F values 


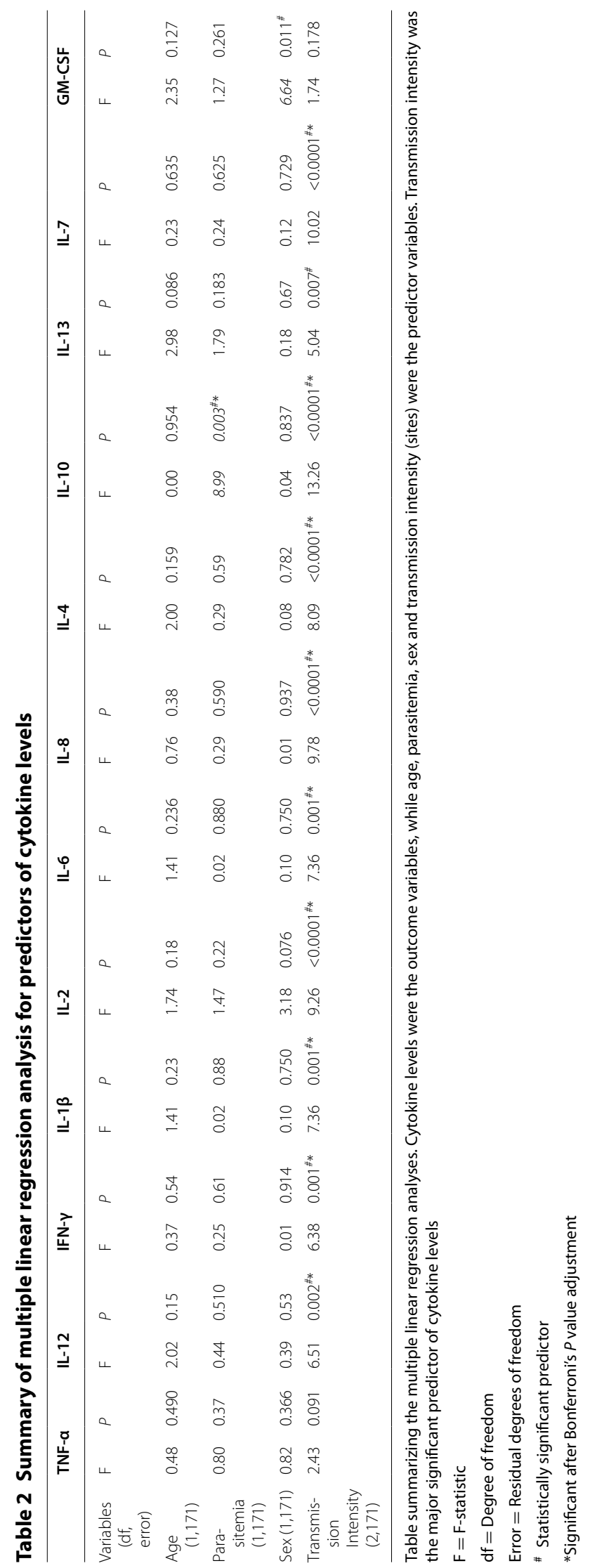




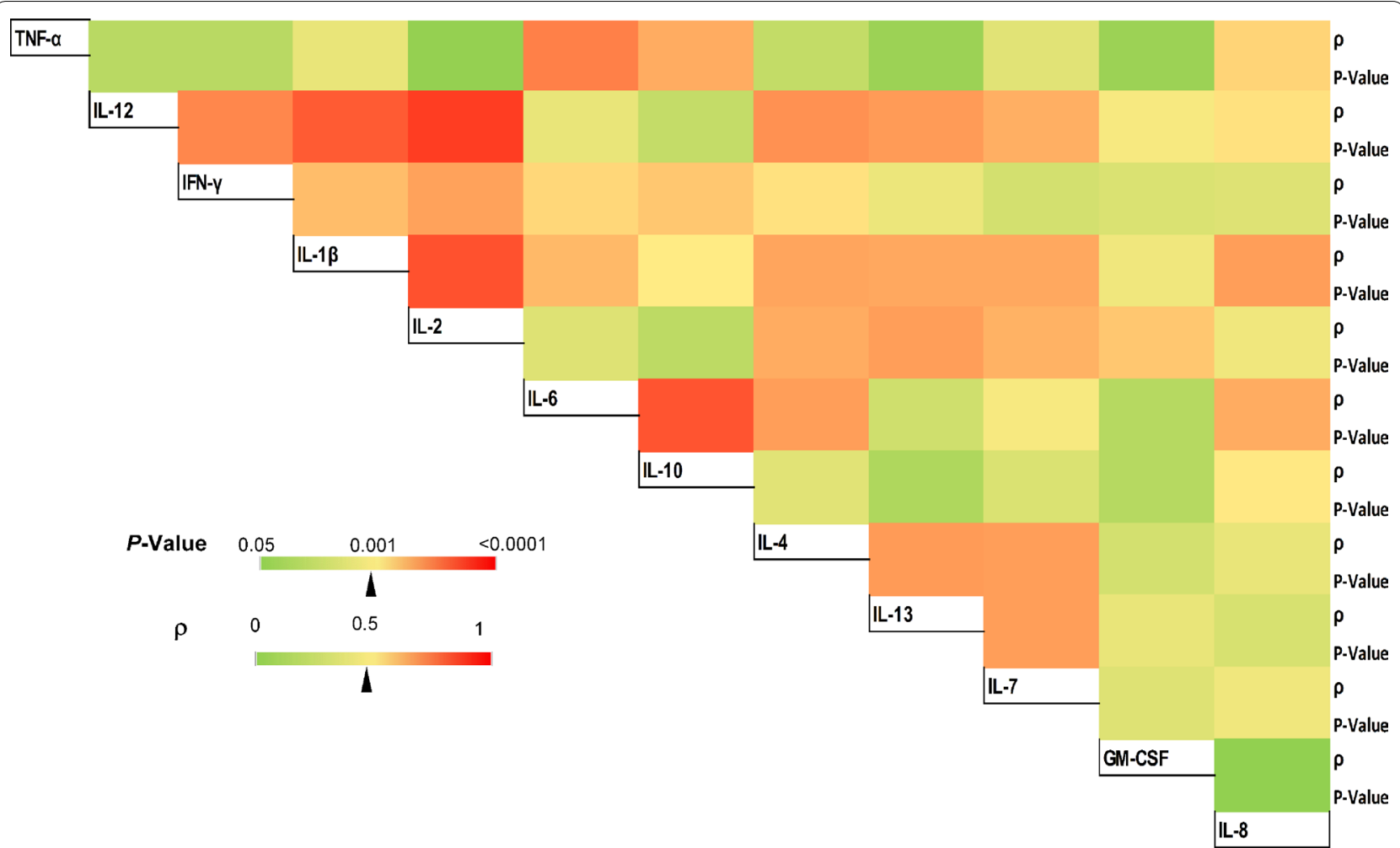

Fig. 5 Correlation matrix showing the relationships between cytokines across the sites. The interrelationships between the levels of inflammatory cytokines in children with malaria were examined by Spearman's correlation test, and the results are summarized in a colour matrix. The strength of correlation between pairs of cytokines are illustrated on a colour scale, where the least statistically significant relationships are coloured green while the most significant are in red. ( $\rho=$ Spearman's correlation coefficient)

in Table 2). Transmission intensity had negative regression coefficients in all the regression models (negative $\beta$-weights, Additional file 4); indicating an inverse relationship with cytokine levels. In addition, the interrelationships among the cytokines across the sites were investigated, and this showed that cytokines generally correlated positively with each other (Fig. 5). Most significantly, the key pro-inflammatory cytokines, including IFN- $\gamma$, IL-12 and IL- 2 strongly correlated with each other, and a strong association was also found between TNF- $\alpha$ and IL-6 (Fig. 5).

\section{Discussion}

Previous studies have established that individuals exposed to endemic malaria transmission can harbour high parasitaemia without clinical symptoms [6, 31, 47], suggesting that the threshold parasitaemia for symptomatic malaria in high transmission areas is higher than that in low-to-medium transmission areas [1, 2, 10, 39]. Data presented here support this phenomenon, whereby increasing transmission intensity was associated with increasing parasite densities in children presenting to hospital with symptomatic malaria. Therefore, it was hypothesized that the regulation of pro-inflammatory responses is a mechanism that accounts for the differences in parasite tolerance in individuals exposed to different transmission intensities. This hypothesis is based on established knowledge that pro-inflammatory responses during infection are characterized by the release of a cascade of soluble immune mediators including cytokines and chemokines that cause fever, and other signs of malaria [48]. The results show that pro-inflammatory responses decreased with increasing transmission intensity (Accra > Navrongo > Kintampo). Consistent with the decreasing levels of pyrogenic cytokines, axillary temperature in the children with malaria decreased with increasing transmission intensity, indicating a decreasing intensity of fever.

Interestingly, significant correlations between parasite density and cytokine levels were observed among children with malaria in Accra only, suggesting that this relationship seems to disappear in higher transmission areas. This assertion was supported by the multiple linear regression analyses, which revealed that transmission intensity was the strongest predictor of cytokine responses during acute malaria infection. These findings 
suggest that higher parasitaemia thresholds for symptomatic malaria in areas of intense malaria transmission may be explained by controlled pro-inflammatory responses, and milder fevers, which consequently delay clinical symptoms until higher parasite densities are attained. On the contrary, lower thresholds of parasitaemia in low transmission areas could be due to a more aggressive pro-inflammatory response against low parasitaemia, leading to more severe fevers and faster onset of clinical manifestation.

High parasitaemia would mean high levels of parasite associated antigens such as glycophosphatidylinositol (GPI) anchors [42, 49, 50], and high levels of damage associated molecular patterns (DAMPs) such as haem from red blood cells [51], which consequently, should induce corresponding high levels of pro-inflammatory response, but such corresponding stimulation was not observed in the high transmission sites. Therefore, tolerance of comparatively higher parasitaemia in areas of intense malaria transmission may be as a result of refractoriness to stimulation from prolonged continuous exposure to parasites and parasite antigens [5, 52]. Previous studies have demonstrated that prolonged stimulation of $\mathrm{CD}^{+}{ }^{+} \mathrm{T}$-cells with high level of antigens mediate adaptive peripheral tolerance, which is characterized by unresponsiveness to further stimulation, with an evident decrease in the secretion of TNF- $\alpha$, IFN- $\gamma$, IL-2, and IL-6 [5, 52-54]. A parallel observation has been described in sepsis, where it was demonstrated that at certain level of stimulation in vitro, cells become refractory to stimulation with bacterial endotoxin, showing no further secretion of pro-inflammatory cytokines [55-57].

In high transmission areas, more frequent infections would mean an almost 'chronic' state of infection [2]. Under this condition, peripheral $\mathrm{CD} 4^{+} \mathrm{T}$-cells are exhausted [58] from persistent stimulation with high levels of parasite associated antigens. In addition, the loss of a $\mathrm{V} \gamma^{+} \delta 2^{+} \mathrm{T}$ cell subset, which rapidly expands and become activated during $P$. falciparum infection, was recently shown to be associated with repeated infections [59]. This sub-set of T-cells has been shown to secrete high levels of TNF- $\alpha$ and IFN- $\gamma$ upon stimulation with iRBCs [60]. Perhaps, low levels of TNF- $\alpha$ and IFN- $\gamma$ observed in the high transmission sites is due to the reduction of this T-cell subset. On the other hand, lower exposure in low transmission areas means that each infection is a separate acute event, which culminates in responses similar to those observed in naïve individuals (i.e., heightened pro-inflammatory response). This result is further buttressed by a recent report which independently demonstrated that pro-inflammatory responses during acute malaria infection increases with decreasing exposure; being highest in naïve adults, followed by immigrants with extended loss of $P$. falciparum exposure, and being lowest in semi-immune individuals residing in an endemic area [61].

Alternatively, there appears to be a mechanism that dampens pro-inflammatory responses [62] in children that have been repeatedly exposed to the parasite [63] through suppression of IL-12 production. Low levels of IL-12 in the high transmission areas could be a result from suppression by ingested haemozoin [64], due to the reported high levels of haemozoin-containing monocytes [65] in children residing in holo-endemic areas. In addition, evidence of suppression of T-cell cytokine responses was recently demonstrated in murine models of malaria [63], where a distinct sub-set of IL-27-secreting Foxp $3^{-} \mathrm{CD} 11 \mathrm{a}^{+} \mathrm{CD} 49 \mathrm{~d}^{+}$malaria antigen-specific $\mathrm{CD} 4^{+}$ T-cells inhibit the production of IL-2, which consequently may dampen IL-12 secretion, resulting in clonal depletion of Th1 cells [63]. Similarly, the development of humoral immune responses appear to be associated with better control of pro-inflammatory responses in children with malaria from Malawi [42].

\section{Conclusion}

Altogether, findings from this study represent significant new knowledge about the mechanisms of malaria pathogenesis and parasite tolerance. The data also provide evidence and understanding of malaria parasite tolerance, an issue of utmost importance in the context of malaria control and eradication since the adverse effects of malaria resurgences are not known. While these findings need to be confirmed by additional investigations of the cellular responses underlying the patterns of cytokine production, data presented here have implications for characterizing the pathophysiology of $P$. falciparum amidst decreasing transmission intensity.

\section{Additional files}

Additional file 1. Assay Sensitivities (minimum detectable concentrations, $\mathrm{pg} / \mathrm{ml}$ ) of analytes.

Additional file 2. The distribution of age and clinical parameters of patients' across the study sites. $(A)$ and $(C)$ Red line across indicates mean while error bars represent standard deviation (One-way ANOVA, with Tukey's posthoc multiple comparison test to reveal pairwise significant differences) (B) Data is presented as a box plot with whiskers and outliers. The box represents the inter-quartile range, while the whiskers represent the 10th and 90th percentiles. The line across the box indicates the median value, closed circles represent outliers (Kruskal-Wallis test, with Dunn's posthoc multiple comparison test to reveal pairwise significant differences).

Additional file 3. Association between cytokines and age across the sites.

Additional file 4. The regression coefficients of transmission intensity as a predictor of cytokine levels. 


\section{Authors' contributions}

GAA conceived the idea, designed the experiments, and supervised the work. TWA performed the experiments in the study. TWA and GAA wrote the paper. YA and KAK contributed to data analysis and editing of the manuscript. All authors read and approved the final manuscript.

\section{Author details}

${ }^{1}$ West African Center for Cell Biology of Infectious Pathogens, Department of Biochemistry, Cell and Molecular Biology, College of Basic and Applied Sciences, University of Ghana, Legon, Accra, Ghana. ${ }^{2}$ Department of Immunology, Noguchi Memorial Institute for Medical Research, College of Health Sciences, University of Ghana, Legon, Accra, Ghana.

\section{Acknowledgements}

The authors thank the parents and children who participated in this study. We are also grateful to the directors, clinical staff, and technicians at LedzokukuKrowor Municipal Assembly Hospital in Accra, War Memorial Hospital in Navrongo, the Navrongo Health Research Centre, the Kintampo Municipal hospital, and the Kintampo Health Research Centre.

\section{Competing interests}

The authors declare that they have no competing interests.

\section{Availability of data and materials}

The datasets generated and/or analysed during the current study are available from the corresponding author on reasonable request.

\section{Consent for publication}

The authors have read and agreed to the content of this manuscript and its publication upon acceptance.

\section{Ethical considerations}

Ethical approvals were obtained from the ethics committees of the Ghana Health Service, Navrongo Health Research Centre, Kintampo Health Research Centre, and Noguchi Memorial Institute for Medical Research, University of Ghana, Accra, Ghana. Participation was voluntary and written informed consent was obtained from parents/guardians of the children.

\section{Funding}

This work was supported by funds from a National Institutes of Health, USA Grant (NIH/NIAID R01Al102848: Awandare) and a DELTAS Africa Grant (DEL15-007: Awandare). Temitope W. Ademolue was supported by a Master's fellowship from a World Bank African Centres of Excellence Grant (ACE02WACCBIP: Awandare). Yaw Aniweh is supported by a postdoctoral fellowship under the DELTAS Africa Grant (DEL-15-007: Awandare). The DELTAS Africa Initiative is an independent funding scheme of the African Academy of Sciences (AAS)'s Alliance for Accelerating Excellence in Science in Africa (AESA) and supported by the New Partnership for Africa's Development Planning and Coordinating Agency (NEPAD Agency) with funding from the Wellcome Trust (107755/Z/15/Z: Awandare), and the UK government. The views expressed in this publication are those of the author(s) and not necessarily those of AAS, NEPAD Agency, Wellcome Trust or the UK government.

\section{Publisher's Note}

Springer Nature remains neutral with regard to jurisdictional claims in published maps and institutional affiliations.

Received: 27 February 2017 Accepted: 1 April 2017

Published online: 11 April 2017

\section{References}

1. Gonçalves BP, Huang C-Y, Morrison R, Holte S, Kabyemela E, Prevots DR, et al. Parasite burden and severity of malaria in Tanzanian children. N Engl J Med. 2014;370:1799-808.

2. Galatas B, Bassat Q, Mayor A. Malaria parasites in the asymptomatic: looking for the hay in the haystack. Trends Parasitol. 2016;32:296-308.
3. Gatton M, Cheng Q. Investigating antigenic variation and other parasitehost interactions in Plasmodium falciparum infections in naïve hosts. Parasitology. 2004;128:367-76.

4. Fowkes FJ, Boeuf P, Beeson JG. Immunity to malaria in an era of declining malaria transmission. Parasitology. 2016;143:139-53.

5. Boutlis CS, Yeo TW, Anstey NM. Malaria tolerance-for whom the cell tolls? Trends Parasitol. 2006:22:371-7.

6. Reyburn H, Mbatia R, Drakeley C, Bruce J, Carneiro I, Olomi R, et al. Association of transmission intensity and age with clinical manifestations and case fatality of severe Plasmodium falciparum malaria. JAMA. 2005:293:1461-70.

7. Osier FH, Fegan G, Polley SD, Murungi L, Verra F, Tetteh KK, et al. Breadth and magnitude of antibody responses to multiple Plasmodium falciparum merozoite antigens are associated with protection from clinical malaria. Infect Immun. 2008;76:2240-8.

8. Rafiou A, Francine C, Ibrahim S, Sonon P, Dechavanne C, Djilali-Saïah A, et al. Plasmodium falciparum infection and age influence parasite growth inhibition mediated by lgG in Beninese infants. Acta Trop. 2016;159:111-9.

9. da Silva HB, de Salles ÉM, Panatieri RH, Boscardin SB, Rodríguez-Málaga SM, Álvarez JM, et al. IFN- $\gamma$-induced priming maintains long-term straintranscending immunity against blood-stage Plasmodium chabaudi malaria. J Immunol. 2013;191:5160-9.

10. Gatton ML, Cheng Q. Evaluation of the pyrogenic threshold for Plasmodium falciparum malaria in naive individuals. Am J Trop Med Hyg. 2002;66:467-73.

11. Gazzinelli RT, Kalantari P, Fitzgerald KA, Golenbock DT. Innate sensing of malaria parasites. Nat Rev Immunol. 2014;14:744-57.

12. loannidis $L J$, Nie $C Q$, Hansen DS. The role of chemokines in severe malaria: more than meets the eye. Parasitology. 2014;141:602-13.

13. Lyke $K$, Burges $R$, Cissoko $Y$, Sangare L, Dao M, Diarra I, et al. Serum levels of the proinflammatory cytokines interleukin-1 beta (IL-1 $\beta), I L-6, I L-8$, IL-10, tumor necrosis factor alpha, and IL-12 (p70) in Malian children with severe Plasmodium falciparum malaria and matched uncomplicated malaria or healthy controls. Infect Immun. 2004;72:5630-7.

14. Clark IA, Alleva LM, Budd AC, Cowden WB. Understanding the role of inflammatory cytokines in malaria and related diseases. Travel Med Infect Dis. 2008;6:67-81.

15. Mackintosh CL, Beeson JG, Marsh K. Clinical features and pathogenesis of severe malaria. Trends Parasitol. 2004;20:597-603.

16. Clark IA, Budd AC, Alleva LM, Cowden WB. Human malarial disease: a consequence of inflammatory cytokine release. Malar J. 2006:5:85.

17. Ing R, Segura M, Thawani N, Tam M, Stevenson MM. Interaction of mouse dendritic cells and malaria-infected erythrocytes: uptake, maturation, and antigen presentation. J Immunol. 2006;176:441-50.

18. Pichyangkul S, Yongvanitchit K, Kum-arb U, Hemmi H, Akira S, Krieg AM, et al. Malaria blood stage parasites activate human plasmacytoid dendritic cells and murine dendritic cells through a Toll-like receptor 9-dependent pathway. J Immunol. 2004;172:4926-33.

19. Awandare GA, Goka B, Boeuf P, Tetteh JK, Kurtzhals JA, Behr C, et al. Increased levels of inflammatory mediators in children with severe Plasmodium falciparum malaria with respiratory distress. J Infect Dis. 2006:194:1438-46.

20. Grau GE, Frei K, Piguet P-F, Fontana A, Heremans H, Billiau A, et al. Interleukin 6 production in experimental cerebral malaria: modulation by anticytokine antibodies and possible role in hypergammaglobulinemia. J Exp Med. 1990;172:1505-8.

21. Looareesuwan S, Sjostrom L, Krudsood S, Wilairatana P, Porter R, Hills F, et al. Polyclonal anti-tumor necrosis factor-alpha Fab used as an ancillary treatment for severe malaria. Am J Trop Med Hyg. 1999;61:26-33.

22. Yoshimoto T, Takahama Y, Wang C-R, Yoneto T, Waki S, Nariuchi H. A pathogenic role of IL-12 in blood-stage murine malaria lethal strain Plasmodium berghei NK65 infection. J Immunol. 1998;160:5500-5.

23. Perkins DJ, Were T, Davenport GC, Kempaiah P, Hittner JB, Ong'echa JM. Severe malarial anemia: innate immunity and pathogenesis. Int J Biol Sci. $2011 \cdot 7 \cdot 1427-42$

24. Cunnington AJ, Riley EM, Walther M. Stuck in a rut? Reconsidering the role of parasite sequestration in severe malaria syndromes. Trends Parasitol. 2013;29:585-92 
25. El-Assaad F, Wheway J, Mitchell AJ, Lou J, Hunt NH, Combes V, et al. Cytoadherence of Plasmodium berghei-infected red blood cells to murine brain and lung microvascular endothelial cells in vitro. Infect Immun. 2013;81:3984-91.

26. Inoue S, Niikura M, Mineo S, Kobayashi F. Roles of IFN-gamma and gammadelta $T$ cells in protective immunity against blood-stage malaria. Front Immunol. 2013;4:258.

27. Shikani HJ, Freeman BD, Lisanti MP, Weiss LM, Tanowitz HB, Desruisseaux MS. Cerebral malaria: we have come a long way. Am J Pathol. 2012;181:1484-92.

28. Rogerson SJ, Hviid L, Duffy PE, Leke RF, Taylor DW. Malaria in pregnancy: pathogenesis and immunity. Lancet Infect Dis. 2007;7:105-17.

29. Boeuf PS, Loizon S, Awandare GA, Tetteh JK, Addae MM, Adjei GO, et al. Insights into deregulated TNF and IL-10 production in malaria: implications for understanding severe malarial anaemia. Malar J. 2012;11:253.

30. Hviid L, Kurtzhals JA, Adabayeri V, Loizon S, Kemp K, Goka BQ, et al. Perturbation and proinflammatory type activation of $V \delta 1+\gamma \delta T$ cells in African children with Plasmodium falciparum malaria. Infect Immun. 2001;69:3190-6.

31. Snow RW, Omumbo JA, Lowe B, Molyneux CS, Obiero J-O, Palmer A, et al. Relation between severe malaria morbidity in children and level of Plasmodium falciparum transmission in Africa. Lancet. 1997:349:1650-4.

32. Awandare GA, Martinson JJ, Were T, Ouma C, Davenport GC, Ong'echa JM, et al. MIF (macrophage migration inhibitory factor) promoter polymorphisms and susceptibility to severe malarial anemia. J Infect Dis. 2009;200:629-37.

33. Smith T, Killeen G, Lengeler C, Tanner M. Relationships between the outcome of Plasmodium falciparum infection and the intensity of transmission in Africa. Am J Trop Med Hyg. 2004;71:80-6.

34. Carneiro I, Roca-Feltrer A, Griffin JT, Smith L, Tanner M, Schellenberg JA, et al. Age-patterns of malaria vary with severity, transmission intensity and seasonality in sub-Saharan Africa: a systematic review and pooled analysis. PLoS ONE. 2010;5:e8988.

35. Owusu-Agyei S, Asante KP, Adjuik M, Adjei G, Awini E, Adams M, et al. Epidemiology of malaria in the forest-savanna transitional zone of Ghana. Malar J. 2009;8:220.

36. Kasasa S, Asoala V, Gosoniu L, Anto F, Adjuik M, Tindana C, et al. Spatiotemporal malaria transmission patterns in Navrongo demographic surveillance site, northern Ghana. Malar J. 2013;12:63.

37. Klinkenberg E, McCall P, Wilson MD, Amerasinghe FP, Donnelly MJ. Impact of urban agriculture on malaria vectors in Accra, Ghana. Malar J. 2008;7:151.

38. Hematology Chanarin I. Principles and procedures. J Clin Pathol. 1984:37:1419.

39. Mensah-Brown HE, Amoako N, Abugri J, Stewart LB, Agongo G, Dickson EK, et al. Analysis of erythrocyte invasion mechanisms of Plasmodium falciparum clinical isolates across 3 malaria-endemic areas in Ghana. J Infect Dis. 2015;212:1288-97.

40. Moncunill G, Aponte JJ, Nhabomba AJ, Dobaño C. Performance of multiplex commercial kits to quantify cytokine and chemokine responses in culture supernatants from Plasmodium falciparum stimulations. PLoS ONE. 2013;8:e52587.

41. Smith TG, Ayi K, Serghides L, McAllister CD, Kain KC. Innate immunity to malaria caused by Plasmodium falciparum. Clin Invest Med. 2002;25:262-72.

42. Mbengue B, Niang B, Niang MS, Varela ML, Fall B, Fall MM, et al. Inflammatory cytokine and humoral responses to Plasmodium falciparum glycosylphosphatidylinositols correlates with malaria immunity and pathogenesis. Immun Inflamm Dis. 2016;4:24-34.

43. do Rosário APF, Lamb T, Spence P, Stephens R, Lang A, Roers A, et al. IL-27 promotes IL-10 production by effector Th1 $\mathrm{CD}^{+} \mathrm{T}$ cells: a critical mechanism for protection from severe immunopathology during malaria infection. J Immunol. 2012;188:1178-90.
44. Medina TS, Costa SP, Oliveira MD, Ventura AM, Souza JM, Gomes TF, et al. Increased interleukin-10 and interferon- $\gamma$ levels in Plasmodium vivax malaria suggest a reciprocal regulation which is not altered by IL-10 gene promoter polymorphism. Malar J. 2011;10:264.

45. Bustinduy AL, Sutherland LJ, Chang-Cojulun A, Malhotra I, DuVall AS, Fairley JK, et al. Age-stratified profiles of serum IL-6, IL-10, and TNF-a cytokines among Kenyan children with Schistosoma haematobium, Plasmodium falciparum, and other chronic parasitic co-infections. Am J Trop Med Hyg. 2015;92:945-51.

46. Peto TJ, Tripura R, Lee SJ, Althaus T, Dunachie S, Nguon C, et al. Association between subclinical malaria infection and inflammatory host response in a pre-elimination setting. PLOS ONE. 2016;11:e0158656.

47. Snow RW, Guerra CA, Noor AM, Myint HY, Hay SI. The global distribution of clinical episodes of Plasmodium falciparum malaria. Nature. 2005:434:214-7.

48. Clark IA, Cowden WB. The pathophysiology of falciparum malaria. Pharmacol Ther. 2003;99:221-60.

49. Schofield L, Hackett F. Signal transduction in host cells by a glycosylphosphatidylinositol toxin of malaria parasites. J Exp Med. 1993;177:145-53.

50. Langhorne J, Ndungu FM, Sponaas A-M, Marsh K. Immunity to malaria: more questions than answers. Nat Immunol. 2008;9:725-32.

51. Mendonca R, Silveira AA, Conran N. Red cell DAMPs and inflammation. Inflamm Res. 2016;65:665-78.

52. Mueller DL. Mechanisms maintaining peripheral tolerance. Nat Immunol. 2010;11:21-7.

53. Singh NJ, Schwartz RH. The strength of persistent antigenic stimulation modulates adaptive tolerance in peripheral CD4 ${ }^{+} \mathrm{T}$ cells. J Exp Med. 2003;198:1107-17.

54. Xing Y, Hogquist KA. T-cell tolerance: central and peripheral. Cold Spring Harbor Perspect Biol. 2012;4:a006957.

55. Cabrera-Perez J, Condotta SA, Badovinac VP, Griffith TS. Impact of sepsis on CD4 T cell immunity. J Leukoc Biol. 2014;96:767-77.

56. West MA, Heagy W. Endotoxin tolerance: a review. Crit Care Med. 2002;30:S64-73.

57. Hotchkiss RS, Monneret G, Payen D. Sepsis-induced immunosuppression: from cellular dysfunctions to immunotherapy. Nat Rev Immunol. 2013;13:862-74.

58. Beltra J-C, Decaluwe H. Cytokines and persistent viral infections. Cytokine. 2016;82:4-15.

59. Jagannathan P, Kim CC, Greenhouse B, Nankya F, Bowen K, Eccles-James I, et al. Loss and dysfunction of $V \delta 2+\gamma \delta T$ cells are associated with clinical tolerance to malaria. Sci Transl Med. 2014;6:251 ra117.

60. Mr Goodier, Lundqvist C, Hammarström ML, Troye-Blomberg M, Langhorne J. Cytokine profiles for human Vy9 + T cells stimulated by Plasmodium falciparum. Parasite Immunol. 1995;17:413-23.

61. Moncunill G, Mayor A, Bardají A, Puyol L, Nhabomba A, Barrios D, et al. Cytokine profiling in immigrants with clinical malaria after extended periods of interrupted exposure to Plasmodium falciparum. PLOS ONE. 2013;8:e73360

62. Urban BC, Ferguson DJ, Pain A, Willcox N, Plebanski M, Austyn JM, et al. Plasmodium falciparum-infected erythrocytes modulate the maturation of dendritic cells. Nature. 1999:400:73-7.

63. Kimura D, Miyakoda M, Kimura K, Honma K, Hara H, Yoshida H, et al. Interleukin-27-producing CD4(+) T cells regulate protective immunity during malaria parasite infection. Immunity. 2016;44:672-82.

64. Keller CC, Yamo O, Ouma C, Ong'echa JM, Ounah D, Hittner JB, et al. Acquisition of hemozoin by monocytes down-regulates interleukin-12 p40 (IL-12p40) transcripts and circulating IL-12p70 through an IL10-dependent mechanism: in vivo and in vitro findings in severe malarial anemia. Infect Immun. 2006;74:5249-60.

65. Awandare GA, Ouma Y, Ouma C, Were T, Otieno R, Keller CC, et al. Role of monocyte-acquired hemozoin in suppression of macrophage migration inhibitory factor in children with severe malarial anemia. Infect Immun. 2007;75:201-10. 